\title{
Who evoked that frame? Some thoughts on context effects and event types
}

\author{
Katrin Erk \\ Department of Linguistics \\ The University of Texas at Austin \\ Austin, Texas 78712 \\ katrin.erk@mail.utexas.edu
}

In memoriam Charles Fillmore, 1929-2014

\begin{abstract}
Lexical substitution is an annotation task in which annotators provide one-word paraphrases (lexical substitutes) for individual target words in a sentence context. Lexical substitution yields a fine-grained characterization of word meaning that can be done by non-expert annotators. We discuss results of a recent lexical substitution annotation effort, where we found strong contextual modulation effects: Many substitutes were not synonyms, hyponyms or hypernyms of the targets, but were highly specific to the situation at hand. This data provides some food for thought for framesemantic analysis.
\end{abstract}

\section{Introduction}

Fillmore (1985) introduces the term "semantics of understanding", or U-semantics. In contrast to the semantics of truth (T-semantics), the goal of Usemantics is to "uncover the nature of the relationship between linguistic texts and the interpreter's full understanding of the texts in their contexts". A central concept of the semantics of understanding is that of the interpretive frames that are necessary for understanding a sentence. Frames are the "coherent schematizations of experience" underlying the words in a given sentence.

This idea of a semantics of understanding, or a frame semantics, has been made concrete in FrameNet (Fillmore et al., 2003), a large lexical database that lists frames for English words and constructions. At this point, it comprises more than 1,100 frames covering more than 12,000 lexical units (LUs), which are pairs of a term and its frame. Researchers working on other languages have adopted the FrameNet idea. Among others, there are now FrameNet resources for Spanish (Subirats and Petruck, 2003), Japanese (Ohara et al., 2004), Italian (Tonelli and Pianta, 2008; Lenci et al., 2010), as well as frame-semantic annotation for German (Erk et al., 2003).

The definition of frames proceeds in a corpusbased fashion, driven by the data (Ellsworth et al., 2004). We stand in this tradition by reporting on a recent annotation effort (Kremer et al., 2014) that collected lexical substitutes for content words in part of the MASC corpus (Ide et al., 2008). If we view substitute sets as indications of the relevant frame, then this data can give us interesting indicators on perceived frames in a naturally occurring text.

\section{Lexical substitution}

The Lexical Substitution task was first introduced in the context of SemEval 2007 (McCarthy and Navigli, 2009). For this dataset, annotators are asked to provide substitutes for a selected word (the target word) in its sentence context - at least one substitute, but possible more, and ideally a single word, though all the datasets contain some multi-word substitutes. Multiple annotators provide substitutes for each target word occurrence. Table 1 shows some examples.

By now, several lexical substitution datasets exist. Some are "lexical sample" datasets, that is, only occurrences of some selected lemmas are annotated (McCarthy and Navigli, 2009; Biemann, 2013), and some are "all-words", providing substitutes for all content words in the given sentences (Sinha and Mihalcea, 2014; Kremer et al., 2014). In addition, there is a cross-lingual lexical substitution dataset (McCarthy et al., 2013), where annotators provided Spanish substitutes for English target words in English sentence context.

Lexical substitution is a method for characterizing word meaning in context that has several attractive properties. Lexical substitution makes it possible to describe word meaning without having to rely on any particular dictionary. In addi- 


\begin{tabular}{l|rr}
\hline relation & verb & noun \\
\hline \hline syn & 12.5 & 7.7 \\
direct-hyper & 9.3 & 7.6 \\
trans-hyper & 2.8 & 4.7 \\
direct-hypo & 11.6 & 8.0 \\
trans-hypo & 3.7 & 3.8 \\
wn-other & 60.7 & 66.5 \\
not-in-wn & 0.9 & 2.2 \\
\hline
\end{tabular}

Table 2: Analysis of lexical substitution data: Relation of the substitute to the target, in percentages by part of speech (from Kremer et al. (2014))

tion, providing substitutes is a task that seems to be well doable by untrained annotators: Both Biemann (2013) and our recent annotation (Kremer et al., 2014) used crowdsourcing to collect the substitutes. ${ }^{1}$

\section{Analyzing lexical substitutes}

In a recent lexical substitution annotation effort (Kremer et al., 2014), we collected lexical substitution annotation for all nouns, verbs, and adjectives in a mixed news and fiction corpus, using untrained annotators via crowdsourcing. The data came from MASC, a freely available part of the American National Corpus that has already been annotated for a number of linguistic phenomena (Ide et al., 2008). All in all, more than 15,000 target tokens were annotated.

After the annotation, we performed a number of analyses in order to better understand the nature of lexical substitutes, by linking substitutes to information on WordNet (Fellbaum, 1998). Among other things, we analyzed the relation between targets and substitutes: Did substitutes tend to be synonyms, hypernyms, or hyponyms or the targets? To classify substitutes, the shortest route from any synset of the target to any synset of the substitute was used. The results are shown in Table 2, for substitutes that are synonyms (syn), hypernyms (direct-hyper, trans-hyper) and hyponyms (direct-hypo, trans-hypo) of the target. The "wn-other" line shows the percentage for substitutes that are in WordNet but not a synonym, hypo- or hypernym of the target, and "not-in-wn"

\footnotetext{
${ }^{1}$ The third example in Table 1 shows that errors do happen: The substitute "accusation" is not appropriate there. Analyses indicate that such errors are rare, though.
}

are substitutes not covered by WordNet. For substitutes that are synonyms, hypernyms, and hyponyms, we see percentages between $8 \%$ and $15 \%$ for both verbs and nouns. We also see that there are few substitutes that are not in WordNet, only $1-2 \%$. Strikingly, $60-66 \%$ of all substitutes are in WordNet, but are "wn-other": neither synonyms nor (transitive) hyponyms or hypernyms of the target. Some of these items can be viewed as missing links in the taxonomy. For example, in the second sentence of Table 2, two of the "wn-other" substitutes of keep are own and possess. But while own and possess are not linked to keep in WordNet, the FrameNet frame RETAINING, which has keep as a lexical unit, inherits from POSSESSION, which has both own and possess as lexical units. But this does not apply to all the "wn-other" substitutes. Some are better explained as effects of contextual modulation, fine-grained meaning distinctions that the sentence context brings about. In the first example in Table 1, there is the possibility that the speaker could be laughing at the other person, and the shoulder-clapping clarifies that this possibility does not correspond to the facts. In the second example in the table, the words possess, enshrine and stage are more specific than the substitutes that are in WordNet, and maybe more appropriate too. In the third example, the word charge has the meaning of dependent, but the situation that the sentence describes suggests that the dependents in questions may be something like underlings or prisoners.

When we look at this data from a framesemantic analysis point of view, the first question that arises is: How specific should the frames be that are listed in FrameNet? For the second example, would we want a very specific "person as precious jewel" frame to be associated with the lexical unit "keep"? From a U-semantics point of view, one could argue that we would in fact want to have this frame, after all: It describes a recognizable abstract situation that is important for the understanding of this sentence. But it does not seem that all "wn-other" cases need to correspond to particular frames of the target word. For example, in the first sentence on Table 1, it does not seem that clarify should be an actual frame involving the word show.

From a computational linguistics point of view, a fine-grained analysis would be necessary in order to correctly predict lexical substitutes like 


\begin{tabular}{ll}
\hline sentence & substitutes \\
\hline \hline I clapped her shoulder to show I was not laughing at her. & $\begin{array}{l}\text { demonstrate, express, establish, indicate, prove, } \\
\text { convey, imply, display, disclose, clarify }\end{array}$ \\
\hline $\begin{array}{l}\text { My fear is that she would live, and I would learn that I had lost her long } \\
\text { before Emil Malaquez translated her into a thing that can be kept, ad- } \\
\text { mired, and loved. }\end{array}$ & $\begin{array}{l}\text { preserve, retain, hold, fix, store, own, possess, } \\
\text { ensine, stage }\end{array}$ \\
$\begin{array}{l}\text { The distinctive whuffle of pleasure rippled through the betas on the } \\
\text { bridge, and Rakal let loose a small growl, as if to caution his charges } \\
\text { against false hope. }\end{array}$ & $\begin{array}{l}\text { dependent, command, accusation, private, com- } \\
\text { pate, ward, junior, underling, enemy, group, } \\
\end{array}$ \\
\hline
\end{tabular}

Table 1: Example from lexical substitution data: Target words underlined, and WordNet-unrelated substitutes shown in italics.

this - but on the other hand, experience with word sense disambiguation has shown that finegrained senses are hard to assign with good accuracy (Palmer et al., 2007).

Another question that this data poses is: What are the items that evoke a frame? That is, what words or phrases in a sentence are responsible that a particular frame becomes important for understanding the sentence? In FrameNet it is a single lemma, multi-word expression or construction that evokes a frame. But one way of looking at the contextual modulation effects in the lexical substitution data is to say that multiple terms or constructions in the context "conspire" to make a frame relevant. In the second sentence of Table 1 , we can point to multiple factors that lead to substitutes like possess and enshrine. There is fact that the THEME of keep is thing, along with the fact that the same thing is being admired and loved, and maybe also the fact that some woman had been translated to said thing. This thought is reminiscent of McRae and colleagues, who study general event knowledge and argue that it is not just verbs that introduce the events, but also arguments (McRae et al., 2005) and combinations of verbs and their arguments (Bicknell et al., 2010).

\section{References}

K. Bicknell, J. Elman, M. Hare, K. McRae, and M. Kutas. 2010. Effects of event knowledge in processing verbal arguments. Journal of Memory and Language, 63(4):489-505.

C. Biemann. 2013. Creating a system for lexical substitutions from scratch using crowdsourcing. Language Resources and Evaluation, 47(1):97-122.

M. Ellsworth, K. Erk, P. Kingsbury, and S. Padó. 2004. PropBank, SALSA and FrameNet: How design determines product. In Proceedings of the LREC Workshop on Building Lexical Resources From Semantically Annotated Corpora, Lisbon, Portugal.
K. Erk, A. Kowalski, S. Padó, and M. Pinkal. 2003. Towards a resource for lexical semantics: A large German corpus with extensive semantic annotation. In Proceedings of ACL, Sapporo, Japan.

C. Fellbaum, editor. 1998. WordNet: An electronic lexical database. MIT Press, Cambridge, MA.

C. J. Fillmore, C. Johnson, and M. Petruck. 2003. Background to framenet. International Journal of Lexicography, 16(3):235-250.

C. J. Fillmore. 1985. Frames and the semantics of understanding. Quaderni di Semantica, 6:222-254.

N. Ide, C.F. Baker, C. Fellbaum, C. Fillmore, and R. Passonneau. 2008. MASC: The manually annotated sub-corpus of American English. In Proceedings of LREC, pages 2455-2461, Marrakech, Morocco.

G. Kremer, K. Erk, S. Padó, and S. Thater. 2014. What substitutes tell us - analysis of an "all-words" lexical substitution corpus. In Proceedings of EACL.

A. Lenci, M. Johnson, and G. Lapesa. 2010. Building an italian framenet through semi-automatic corpus analysis. In Proceedings of LREC, La Valletta.

D. McCarthy and R. Navigli. 2009. The English lexical substitution task. Language Resources and Evaluation, 43(2):139-159.

D. McCarthy, R. Sinha, and R. Mihalcea. 2013. The cross-lingual lexical substitution task. Language Resources and Evaluation, 47(3):607-638.

K. McRae, M. Hare, J. L. Elman, and T. Ferretti. 2005. A basis for generating expectancies for verbs from nouns. Memory \& Cognition, 33(7):1174-1184.

K. Ohara, S. Fujii, T. Ohori, R. Suzuki, H. Saito, and S. Ishizaki. 2004. The Japanese FrameNet project: An introduction. In Proceedings of the LREC Workshop on Building Lexical Resources from Semantically Annotated Corpora, Lisbon, Portugal.

M. Palmer, H. Trang Dang, and C. Fellbaum. 2007. Making fine-grained and coarse-grained sense distinctions, both manually and automatically. Natural Language Engineering, 13:137-163. 
R. Sinha and R. Mihalcea. 2014. Explorations in lexical sample and all-words lexical substitution. Natural Language Engineering, 20(1):99-129.

C. Subirats and M.R.L. Petruck. 2003. Surprise! Spanish FrameNet! In Proceedings of the Workshop on Frame Semantics, XVII. International Congress of Linguists, Prague, Czech Republic.

S. Tonelli and E. Pianta. 2008. Frame information transfer from english to italian. In Proceedings of LREC, Marrakech, Morocco. 